\title{
The bargaining set of a large economy with differential information $\star$
}

\section{Ezra Einy $^{1}$, Diego Moreno ${ }^{2}$, and Benyamin Shitovitz ${ }^{3}$}

1 Department of Economics, Ben Gurion University of the Negev, Beer Sheva, 84105 ISRAEL (e-mail: einy@bgumail bgu ac il)

2 Departamento de Economía, Universidad Carlos III de Madrid, 28903 Getafe, SPAIN (e-mail: dmoreno@eco uc3m es)

3 Department of Economics, University of Haifa, Haifa, 31905 ISRAEL (e-mail: binya@econ haifa ac il)

Received: February 15, 1999; revised version: August 9, 1999

Summary. We study the Mas-Colell bargaining set of an exchange economy with differential information and a continuum of traders. We established the equivalence of the private bargaining set and the set of Radner competitive equilibrium allocations. As for the weak fine bargaining set, we show that it contains the set of competitive equilibrium allocations of an associated symmetric information economy in which each trader has the "joint information" of all the traders in the original economy, but unlike the weak fine core and the set of fine value allocations, it may also contain allocations which are not competitive in the associated economy.

Keywords and Phrases: General equilibrium in large exchange economies with differential information, Weak fine bargaining set, Core, Value.

JEL Classification Numbers: D50, D82, C70.

\section{Introduction}

There is a large literature studying the cooperative foundations of competitive equilibria. In this literature the core is introduced as a solution concept that, without appealing to an specific institutional framework, identifies the allocations that may result from a multilateral bargaining situation in which traders discuss alternative mutually beneficial trades. A difficulty of the core as a solution concept is that when a coalition threats to break an agreement it does not take into account

* We thank the associate editor and a referee for helpful comments This work was done while Einy and Shitovitz visited the Department of Economics of the Universidad Carlos III de Madrid Einy acknowledges the financial support of the Universidad Carlos III de Madrid Moreno acknowledges the support of the Spanish Ministry of Education (DGES), grant PB97-0091 Shitovitz acknowledges the support of the Spanish Ministry of Education, grant SAB98-0059

Correspondence to: D Moreno 
how other coalitions may react to this threat; i.e., coalitional objections to a proposed allocation are not required to be robust to possible counterobjections. To address this issue, Aumann and Maschler (1964) introduce the notion of bargaining set for cooperative games with finitely many players. In the definition of the bargaining set, coalitional objections to a proposed agrement that admit counterobjections are disregarded; that is, when demanding improvements, coalitions must take account of the reactions of other coalitions-for a discussion of this issue (see Maschler, 1976, 1992). Mas-Colell (1989) introduces a new notion of bargaining set and shows that in a complete information exchange economy with a continuum of traders it coincides with the set of competitive allocations. [The equivalence of the core, the set of value allocations and the set of competitive equilibrium allocations in this context was established by Aumann $(1964,1975)$.]

Radner $(1968,1982)$ introduces a model of exchange economy with differential information in which every trader is characterized by a state dependent utility function, a random initial endowment, an information partition, and a prior belief. In this framework, traders arrange contingent contracts for trading commodities before they obtain any information about the realized state of nature. Radner (1968) extends the notion of Arrow-Debreu competitive equilibrium to this model. In the definition of competitive equilibrium (in the sense of Radner), the information of an agent places a restriction on his feasible trades (i.e., his budget set): better information allows for more contingent trades (i.e., enlarges the agent's budget set). Thus, a Radner competitive equilibrium rewards the information advantage of a trader.

In this paper we study the relation of the Mas-Colell bargaining set and the set of competitive allocations of an economy with differential information and a continuum of traders. Our aim is not only to determine whether there are equivalence results similar to those found for complete information economies, but also to explore whether the bargaining set discriminates between traders with differential information.

In the context of exchange economies with differential information and finitely many traders, Yannelis (1991) introduces the concept of private core, and proves that it is non-empty. Krasa and Yannelis (1994) introduce the notion of private value allocation, and discuss examples where the private value rewards the information advantage of a trader. In this approach, the traders of a coalition use only their private information (i.e., there is no information exchange). Einy, Moreno and Shitovitz (1998) show that in a Radner type economy with a continuum of traders the private core coincides with the set of Radner competitive equilibrium allocations, and Einy and Shitovitz (1998) establish the analogous result for the set of private value allocations. Thus, as pointed out by Koutsougeras and Yannelis (1993) and Krasa and Yannelis (1994), the private core and private value reward the information advantage of a trader.

Our findings in the present paper confirm these results: we introduce the notion of Mas-Colell private bargaining set, and we show that in a Radner type economy with a continuum of traders this set coincides with the set of Radner competitive allocations. Our proof that the Mas-Colell private bargaining set 
coincides with the set of Radner competitive equilibrium allocations is along the lines of the proof of Mas-Colell (1989), although the details of some of the arguments require more involved constructions because we must deal with the measurability restrictions imposed by the traders differential information, and also with the possibility that competitive prices may not be strictly positive.

An interesting question is whether the information advantage of a trader is rewarded when we account for the possibility that traders in a coalition may communicate and share some of their information. These possibilities are captured by the notion of fine core due to Wilson (1978). Einy, Moreno and Shitovitz (1998) show that the set of (weak) fine core allocations of a Radner type economy with a continuum of traders coincides with set of competitive equilibrium allocations of an associated symmetric information economy in which each trader has the "joint information" of all the traders in the original economy. Einy, Moreno and Shitovitz (1999) establish an analogous result for fine value allocations.

These results suggest that when the possibility of sharing information is introduced the information advantage of a trader is worthless. Interestingly, this is not the case when we use the weak fine bargaining set as the solution concept: we find that in a Radner type economy with a continuum of traders the weak fine bargaining set contains the competitive allocations of the associated symmetric information economy, albeit it may also contain other allocations where the traders with an information advantage are more favorably treated. Thus, in contrast with the weak fine core and the set of weak fine value allocations, the weak fine bargaining set may reward the information advantage of a trader.

\section{The model}

We consider a Radner-type exchange economy $\mathscr{E}$ with differential information (e.g., Radner $(1968,1982))$.

The space of traders is a measure space $(T, \Sigma, \mu)$, where $T$ is a set (the set of traders), $\Sigma$ is a $\sigma$-field of subsets of $T$ (the set of coalitions), and $\mu$ is a non-atomic measure on $\Sigma$. The commodity space is $\Re_{+}^{l}$. The space of states of nature is a finite set $\Omega$. The economy extends over two time periods, $\tau=0,1$. Consumption takes place at $\tau=1$. At $\tau=0$ there is uncertainty over the state of nature; in this period traders arrange contracts that may be contingent on the realized state of nature at $\tau=1$. At $\tau=1$ traders do not necessarily know which state of nature $\omega \in \Omega$ actually occurred, although they know their own endowments, and may also have some additional information about the state of nature. We do not assume, however, that traders know their own utility function.

The information of a trader $t \in T$ is described by a partition $\Pi_{t}$ of $\Omega$. We denote by $\mathscr{T}_{t}$ the field generated by $\Pi_{t}$. If $\omega_{0}$ is the true state of nature, at $\tau=1$ trader $t$ observes the member of $\Pi_{t}$ which contains $\omega_{0}$. Every trader $t \in T$ has a probability distribution $q_{t}$ on $\Omega$ which represents his prior beliefs. The preferences of a trader $t \in T$ are represented by a state dependent utility function, $u_{t}: \Omega \times \Re_{+}^{l} \rightarrow \Re$ such that for every $(t, x) \in \Omega \times \Re_{+}^{l}$, the mapping 
$(t, x) \rightarrow u_{t}(\omega, x)$ is $\Sigma \times \mathscr{B}$ measurable, where $\omega$ is a fixed member of $\Omega$, and $\mathscr{B}$ is the $\sigma$-field of Borel subsets of $\Re_{+}^{l}$. If $x$ is a random bundle (i.e., a function from $\Omega$ to $\Re_{+}^{l}$ ) we denote by $h_{t}(x)$ the expected utility of trader $t \in T$ from $x$. That is

$$
h_{t}(x)=\sum_{\omega \in \Omega} q_{t}(\omega) u_{t}(\omega, x(\omega)) .
$$

An assignment is a function $\mathbf{x}: T \times \Omega \rightarrow \Re_{+}^{l}$ such that for every $\omega \in \Omega$ the function $\mathbf{x}(\cdot, \omega)$ is $\mu$-integrable on $T$. There is a fixed initial assignment $\mathbf{e} ; \mathbf{e}(t, \omega)$ represents the initial endowment of trader $t \in T$ in the state of nature $\omega \in \Omega$. We assume that $\mathbf{e}(t, \omega)$ is in $\Re_{++}^{l}$ for every $(t, \omega) \in T \times \Omega$, and for every $t \in T$ the function $\mathbf{e}(t, \cdot)$ is $\mathscr{T}_{t}$-measurable.

In the rest of the paper, an economy $\mathscr{E}$ is an atomless economy with differential information as described above. Also we use the following notation: For two vectors $x=\left(x_{1}, \ldots, x_{l}\right)$ and $y=\left(y_{1}, \ldots, y_{l}\right)$ in $\Re^{l}$ we write $x \geq y$ when $x_{k} \geq y_{k}$ for all $1 \leq k \leq l, x>y$ when $x \geq y$ and $x \neq y$, and $x \gg y$ when $x_{k}>y_{k}$ for all $1 \leq k \leq l$.

Let $\mathscr{E}$ be an economy. A private allocation is an assignment $\mathbf{x}$ such that

(2.1) for almost all $t \in T$ the function $\mathbf{x}(t, \cdot)$ is $\mathscr{T}_{t}$-measurable, and

(2.2) $\int_{T} \mathbf{x}(t, \omega) d \mu \leq \int_{T} \mathbf{e}(t, \omega) d \mu$ for all $\omega \in \Omega$.

A price system is a non-zero function $p: \Omega \rightarrow \Re_{+}^{l}$. Let $t \in T$. Write $M_{t}$ for the set of all $\mathscr{T}_{t}$-measurable functions from $\Omega$ to $\Re_{+}^{l}$. For a price system $p$, define the budget set of $t$ by

$$
B(p, t)=\left\{x \mid x \in M_{t} \text { and } \sum_{\omega \in \Omega} p(\omega) \cdot x(\omega) \leq \sum_{\omega \in \Omega} p(\omega) \cdot \mathbf{e}(t, \omega)\right\} .
$$

A competitive equilibrium (in the sense of Radner) is a pair $(p, \mathbf{x})$ where $p$ is a price system and $\mathbf{x}$ is private allocation such that

(2.3) for almost all $t \in T$ the function $\mathbf{x}(t, \cdot)$ maximizes $h_{t}$ on $B(p, t)$, and

$\sum_{\omega \in \Omega} p(\omega) \cdot \int_{T} \mathbf{x}(t, \omega) d \mu=\sum_{\omega \in \Omega} p(\omega) \cdot \int_{T} \mathbf{e}(t, \omega) d \mu$

A competitive allocation is a private allocation $\mathbf{x}$ for which there exists a price system $p$ such that $(p, \mathbf{x})$ is a competitive equilibrium.

Note that since $\Omega$ is a finite set there is a finite family $\left\{\Pi_{i}\right\}_{i=1}^{n}$ of partitions of $\Omega$ such that for all $t \in T$ there is $1 \leq i \leq n$ with $\Pi_{t}=\Pi_{i}$. We assume that for all $1 \leq i \leq n$, the set $T_{i}=\left\{t \in T \mid \Pi_{t}=\Pi_{i}\right\}$ is measurable, and $\mu\left(T_{i}\right)>0$. For all $1 \leq i \leq n$ we denote by $\mathscr{T} i$ the field generated by $\Pi_{i}$.

Throughout the paper we assume that for all $t \in T$ and $\omega \in \Omega$ the function $u_{t}(\omega, \cdot)$ is strictly increasing and continuous on $\Re_{+}^{l}$. (A function $u: \Re_{+}^{l} \rightarrow \Re$ is strictly increasing if for all $x, y \in \Re_{+}^{l}, x>y$ implies $u(x)>u(y)$.)

Einy, Moreno and Shitovitz (1998) have shown that if the utility functions of the traders are continuous and strictly increasing, and if every commodity is present in the market (i.e., $\int_{T} \mathbf{e}(t, \omega) d \mu \gg 0$ for all $\omega \in \Omega$ ), then a competitive 
equilibrium (in the sense of Radner) exists when the economy is irreducible (see Theorem A in Einy, Moreno and Shitovitz, 1998). Since in our model the initial endowments of the traders are in $\Re_{++}^{l}$, the economies we consider here are irreducible (see Proposition 3.1 in Einy, Moreno and Shitovitz, 1998), and therefore always have a competitive equilibrium.

\section{The private bargaining set}

In this section we introduce the notion of (Mas-Colell) private bargaining set, and show that it coincides with the set of (Radner) competitive allocations. We begin by extending to our model the definition of private core due to Yannelis (1991).

Let $\mathscr{E}$ be an economy, and let $\mathbf{x}$ be a private allocation. A private objection to $\mathbf{x}$ is a pair $(S, \mathbf{y})$ such that

(3.1) $\mu(S)>0$,

(3.2) $\mathbf{y}(t, \cdot)$ is $\mathscr{T}_{t}$-measurable for almost all $t \in S$,

(3.3) $\int_{S} \mathbf{y}(t, \omega) d \mu \leq \int_{S} \mathbf{e}(t, \omega)$ for all $\omega \in \Omega$,

(3.4) $h_{t}(\mathbf{y}(t, \cdot)) \geq h_{t}(\mathbf{x}(t, \cdot))$ for almost all $t \in S$, and

(3.5) $\mu\left(\left\{t \in S \mid h_{t}(\mathbf{y}(t, \cdot))>h_{t}(\mathbf{x}(t, \cdot))\right\}\right)>0$.

An assignment $\mathbf{x}$ is a private core allocation of $\mathscr{E}$ if it has no private objection. The private core of $\mathscr{E}$ is the set of all private core allocations of $\mathscr{E}$.

In defining the core, usually the inequalities (3.4) are strict, and (3.5) is omitted. Since in our framework the utility functions of the traders are continuous and strictly increasing in every state of nature, these alternative definitions of the core are equivalent.

Let $\mathscr{E}$ be an economy, let $\mathbf{x}$ be a private allocation and let $(S, \mathbf{y})$ be a private objection to $\mathbf{x}$. A private counterobjection to $(S, \mathbf{y})$ is a pair $(Q, \mathbf{z})$ such that

(3.6) $\mu(Q)>0$,

(3.7) $\mathbf{z}(t, \cdot)$ is $\widetilde{F_{t}}$-measurable for almost all $t \in Q$,

(3.8) $\int_{Q} \mathbf{z}(t, \omega) d \mu \leq \int_{Q} \mathbf{e}(t, \omega)$ for all $\omega \in \Omega$,

(3.9) $h_{t}(\mathbf{z}(t, \cdot))>h_{t}(\mathbf{y}(t, \cdot))$ for almost all $t \in Q \cap S$, and

(3.10) $h_{t}(\mathbf{z}(t, \cdot))>h_{t}(\mathbf{x}(t, \cdot))$ for almost all $t \in Q \backslash S$.

A private objection to $\mathbf{x},(S, \mathbf{y})$, is justified if it has no private counterobjection. The (Mas-Colell) private bargaining set is the set of private allocations which have no justified private objection. Note that the private core of an economy $\mathscr{E}$ is a subset of the private bargaining set of $\mathscr{E}$.

Theorem A. The private bargaining set of an economy $\mathscr{E}$ coincides with the set of Radner competitive allocations of $\mathscr{E}$. 
Einy, Moreno and Shitovitz (1998) have established that the set of Radner competitive equilibrium allocations of an economy $\mathscr{E}$ as defined here coincides with the private core of $\mathscr{E}$. Since the private core is a subset of the private bargaining set, in order to prove Theorem A it suffices to show that every private bargaining set allocation of $\mathscr{E}$ is a competitive allocation of $\mathscr{E}$. Our proof of this result is along the lines of the proof of Theorem 1 in Mas-Colell (1989), although the details of some of the arguments require more involved constructions because we must deal with the measurability restrictions imposed by the traders differential information, and also with the possibility that competitive prices may not be strictly positive. (In spite of the fact that traders utility functions are strictly increasing, in an economy with differential information we cannot guarantee that competitive prices are strictly positive.) In establishing this result, the notion of competitive objection will be useful.

A private objection $(S, \mathbf{y})$ to $\mathbf{x}$ is a competitive objection if there is a price system $p$ such that for almost all $t \in T$

(3.11) if $t \in S$ and $z \in M_{t}$ satisfies $h_{t}(z) \geq h_{t}(\mathbf{y}(t, \cdot))$, then $\sum_{\omega \in \Omega} p(\omega) \cdot z(\omega) \geq$ $\sum_{\omega \in \Omega} p(\omega) \cdot \mathbf{e}(t, \omega)$, and

(3.12) if $t \in T \backslash S$ and $z \in M_{t}$ satisfies $h_{t}(z) \geq h_{t}(\mathbf{x}(t, \cdot))$, then

$\sum_{\omega \in \Omega} p(\omega) \cdot z(\omega) \geq \sum_{\omega \in \Omega} p(\omega) \cdot \mathbf{e}(t, \omega)$.

Theorem A is a consequence of the following two lemmata.

Lemma 3.1. Every competitive objection $(S, \mathbf{y})$ to a private allocation $\mathbf{x}$ is justified.

Proof. Let $(S, \mathbf{y})$ be competitive objection to an allocation $\mathbf{x}$, and let $p$ be the price system associated with $(S, \mathbf{y})$. Assume contrary to our claim that there is a private counterobjection $(Q, \mathbf{z})$ to $(S, \mathbf{y})$. Then $h_{t}(\mathbf{z}(t, \cdot))>h_{t}(\mathbf{y}(t, \cdot))$ for almost all $t \in Q \cap S$, and $h_{t}(\mathbf{z}(t, \cdot))>h_{t}(\mathbf{x}(t, \cdot))$ for almost all $t \in Q \backslash S$. Since for all $t \in T$ and all $\omega \in \Omega, u_{t}(\omega, \cdot)$ is strictly increasing and $\mathbf{e}(t, \omega) \gg 0$, and since $(S, \mathbf{y})$ is a competitive objection, for almost all $t \in Q$ we have

$$
\sum_{\omega \in \Omega} p(\omega) \cdot \mathbf{z}(t, \omega)>\sum_{\omega \in \Omega} p(\omega) \cdot \mathbf{e}(t, \omega) .
$$

This contradicts that for all $\omega \in \Omega, \int_{Q} \mathbf{z}(t, \omega) d \mu \leq \int_{Q} \mathbf{e}(t, \omega) d \mu$.

Lemma 3.2. If $\mathbf{x}$ is not a competitive allocation, then there is a competitive objection to $\mathbf{x}$.

Proof. Throughout the proof we assume without loss of generality that $\mu(T)=$ 1. Assume that $\mathbf{x}$ is not a competitive allocation. We construct a competitive objection to $\mathbf{x}$. Define

$$
P=\bigcap_{i=1}^{n}\left\{p \in\left(\Re_{+}^{l}\right)^{\Omega} \mid \sum_{\omega \in \Omega} \sum_{j=1}^{l} p_{j}(\omega)=1 \text { and } \sum_{\omega \in A} p(\omega) \gg 0, \text { for all } A \in \Pi_{i}\right\} .
$$


Then $P$ is a non-empty convex subset of $\left(\Re_{+}^{l}\right)^{\Omega}$. Now for $p \in P$ and $t \in T$, the budget set $B(p, t)$ is a compact subset of $M_{t}$. Therefore the function $h_{t}$ attains a maximum on $B(p, t)$. For all $p \in P$ and all $t \in T$ let

$$
D(p, t)=\left\{x \in M_{t} \mid x \text { maximizes } h_{t} \text { on } B(p, t)\right\},
$$

and

$$
F(p, t)=\left\{\begin{array}{lll}
D(p, t) & \text { if } & h_{t}(D(p, t))>h_{t}(\mathbf{x}(t, \cdot)) \\
D(p, t) \cup\{\mathbf{e}(t, \cdot)\} & \text { if } & h_{t}(D(p, t))=h_{t}(\mathbf{x}(t, \cdot)) \\
\{\mathbf{e}(t, \cdot)\} & \text { if } & h_{t}(D(p, t))<h_{t}(\mathbf{x}(t, \cdot))
\end{array}\right.
$$

Let

$$
\alpha=1+\sum_{\omega \in \Omega} \sum_{j=1}^{l} \int_{T} e_{j}(t, \omega) d \mu,
$$

and let

$$
K=\left\{x \in\left(\Re_{+}^{l}\right)^{\Omega} \mid \sum_{\omega \in \Omega} \sum_{j=1}^{l} x_{j}(\omega) \leq \alpha\right\},
$$

and

$$
\hat{K}=\left\{x \in K \mid \sum_{\omega \in \Omega} \sum_{j=1}^{l} x_{j}(\omega)=\alpha\right\} .
$$

Note that $K$ is a non-empty compact convex subset of $\left(\Re_{+}^{l}\right)^{\Omega}$. Write $\bar{P}$ for the closure of $P$, and define a correspondence $\phi: \bar{P} \times T \rightarrow 2^{K}$ by

$$
\phi(p, t)= \begin{cases}F(p, t) \cap K & \text { if } \quad p \in P \text { and } D(p, t) \cap K \neq \emptyset \\ \hat{K} \cap\{\lambda d \mid d \in F(p, t), \lambda \geq 0\} & \text { if } \quad p \in P \text { and } D(p, t) \cap K=\emptyset \\ B(p, t) \cap \hat{K} & \text { if } \quad p \in \bar{P} \backslash P .\end{cases}
$$

For every $p \in \bar{P}$ define

$$
\psi(p)=\int_{T} \phi(p, t) d \mu-\int_{T} \mathbf{e}(t, \cdot) d \mu .
$$

Then for every $p \in \bar{P}, \psi(p)$ is a non-empty convex subset of the compact convex set $K$. The proof that $\psi$ is also upper semicontinuous on $\bar{P}$ is standard. From the definition of $\psi$ it is clear that for all $p \in \bar{P}$ we have $p \cdot \psi(p) \leq 0$. Therefore by (1) in Section 5.6 of Debreu (1959), there exists $p^{*} \in \bar{P}$ and $z^{*} \in \psi\left(p^{*}\right)$ such that $z^{*} \leq 0$. We show that $p^{*} \notin \bar{P} \backslash P$. Suppose $p^{*} \in \bar{P} \backslash P$; then $z^{*} \in$ $\left(\int_{T}\left(B\left(p^{*}, t\right) \cap \hat{K}\right) d \mu-\int_{T} \mathbf{e}(t, \cdot) d \mu\right)$. Therefore

$$
\sum_{\omega \in \Omega} \sum_{j=1}^{l} z_{j}^{*}(\omega)=\alpha-\sum_{\omega \in \Omega} \sum_{j=1}^{l} \int_{T} e_{j}(t, \omega) d \mu=1,
$$

which contradicts $z^{*} \leq 0$. Thus $p^{*} \notin \bar{P} \backslash P$. As $z^{*} \leq 0$, we have

$$
z^{*} \in \int_{T}\left(F\left(p^{*}, t\right) \cap K\right) d \mu-\int_{T} \mathbf{e}(t, \cdot) d \mu .
$$


Hence there exists an integrable function $f$ on $T$ such that $f(t) \in F\left(p^{*}, t\right)$ for all $t \in T$ and $z^{*}=\int_{T} f(t) d \mu-\int_{T} \mathbf{e}(t, \cdot) d \mu$. Write

$$
S=\left\{t \in T \mid f(t) \in D\left(p^{*}, t\right)\right\},
$$

and

$$
C\left(p^{*}\right)=\left\{t \in T \mid h_{t}\left(D\left(p^{*}, t\right)\right)>h_{t}(\mathbf{x}(t, \cdot))\right\} .
$$

Since $\mathbf{x}$ is not a competitive allocation, we have $\mu\left(C\left(p^{*}\right)\right)>0$. As $C\left(p^{*}\right) \subset S$, $\mu(S)>0$.

Now for all $(t, \omega) \in T \times \Omega$ let

$$
\mathbf{y}(t, \omega)=(f(t))(\omega)
$$

We show that $(S, \mathbf{y})$ is a competitive objection to $\mathbf{x}$. As noted above, $\mu(S)>0$. Since $z^{*} \leq 0$ and $f(t)=\mathbf{e}(t, \cdot)$ for $t \in T \backslash S$, we have

$$
\int_{S} \mathbf{y}(t, \omega) d \mu \leq \int_{S} \mathbf{e}(t, \omega) d \mu,
$$

for all $\omega \in \Omega$. By the definition of $\mathbf{y}$ we have $h_{t}(\mathbf{y}(t, \cdot)) \geq h_{t}(\mathbf{x}(t, \cdot))$ for all $t \in S$, and $h_{t}(\mathbf{y}(t, \cdot))>h_{t}(\mathbf{x}(t, \cdot))$ for all $t \in C\left(p^{*}\right)$. If $t \in S$ and $z \in M_{t}$ satisfies $h_{t}(z) \geq h_{t}(\mathbf{y}(t, \cdot))$, then $h_{t}\left(D\left(p^{*}, t\right)\right) \leq h_{t}(z)$. Therefore

$$
\sum_{\omega \in \Omega} p^{*}(\omega) \cdot z(\omega) \geq \sum_{\omega \in \Omega} p^{*}(\omega) \cdot \mathbf{e}(t, \omega) .
$$

Let $t \in T \backslash S$. Then $h_{t}\left(D\left(p^{*}, t\right)\right) \leq h_{t}(\mathbf{x}(t, \cdot))$. Therefore if $z \in M_{t}$ satisfies $h_{t}(z) \geq h_{t}(\mathbf{x}(t, \cdot))$, then $h_{t}(z) \geq h_{t}\left(D\left(p^{*}, t\right)\right)$, and thus

$$
\sum_{\omega \in \Omega} p^{*}(\omega) \cdot z(\omega) \geq \sum_{\omega \in \Omega} p^{*}(\omega) \cdot \mathbf{e}(t, \omega) .
$$

This completes the proof that $(S, \mathbf{y})$ is a competitive objection to $\mathbf{x}$.

\section{The weak fine bargaining set}

In this section we introduce the notion of weak fine bargaining set and study its relation with the set of competitive allocations.

Let $\mathscr{E}$ be an economy, and let $S \in \Sigma$. Define

$$
I(S)=\left\{i \mid 1 \leq i \leq n \text { and } \mu\left(S \cap T_{i}\right)>0\right\} .
$$

where $n$ and $T_{i}$ are defined in Section 2. A weak fine allocation is an assignment x such that

(4.1) For all $t \in T, \mathbf{x}(t, \cdot)$ is $\bigvee_{i=1}^{n} \mathscr{T}_{i}$-measurable, and

(4.2) $\int_{T} \mathbf{x}(t, \omega) d \mu \leq \int_{S} \mathbf{e}(t, \omega)$ for all $\omega \in \Omega$. 
Let $\mathbf{x}$ be a weak fine allocation. A weak fine objection to $\mathbf{x}$ is a pair $(S, \mathbf{y})$ such that

(4.3) $\mu(S)>0$,

(4.4) $\mathbf{y}(t, \cdot)$ is $\bigvee_{i \in I(S)} \mathscr{T}_{i}$-measurable for all $t \in S$,

(4.5) $\int_{S} \mathbf{y}(t, \omega) d \mu \leq \int_{S} \mathbf{e}(t, \omega)$ for all $\omega \in \Omega$,

(4.6) $h_{t}(\mathbf{y}(t, \cdot)) \geq h_{t}(\mathbf{x}(t, \cdot))$ for almost all $t \in S$, and

(4.7) $\mu\left(\left\{t \in S \mid h_{t}(\mathbf{y}(t, \cdot))>h_{t}(\mathbf{x}(t, \cdot))\right\}\right)>0$.

A weak fine core allocation of $\mathscr{E}$ is a weak fine allocation $\mathbf{x}$ which has no weak fine objection. The weak fine core of $\mathscr{E}$ is the set of all weak fine core allocations of $\mathscr{E}$.

The weak fine core was introduced in Yannelis (1991), Allen (1991), and Koutsougeras and Yannelis (1993). In order to define the weak fine bargaining set we need to introduce the definition of weak fine counterobjection.

Let $\mathscr{E}$ be an economy, let $\mathbf{x}$ be a weak fine allocation, and let $(S, \mathbf{y})$ be a weak fine objection to $\mathbf{x}$. A weak fine counterobjection to $(S, \mathbf{y})$ is a pair $(Q, \mathbf{z})$ such that

(4.8) $\mu(Q)>0$,

(4.9) $\mathbf{z}(t, \cdot)$ is $\bigvee_{i \in I(Q)} \mathscr{T}_{i}$-measurable for almost all $t \in Q$,

(4.10) $\int_{Q} \mathbf{z}(t, \omega) d \mu \leq \int_{Q} \mathbf{e}(t, \omega)$ for all $\omega \in \Omega$,

(4.11) $h_{t}(\mathbf{z}(t, \cdot))>h_{t}(\mathbf{y}(t, \cdot))$ for almost all $t \in Q \cap S$, and

(4.12) $h_{t}(\mathbf{z}(t, \cdot))>h_{t}(\mathbf{x}(t, \cdot))$ for almost all $t \in Q \backslash S$.

A weak fine objection $(S, \mathbf{y})$ to a weak fine allocation $\mathbf{x}$ is justified if it has not weak fine counterobjection. The (Mas-Colell) weak fine bargaining set is the set of weak fine allocations which have no justified weak fine objection.

Let $\mathscr{E}$ be an economy. Denote by $\mathscr{E}^{*}$ the economy obtained from $\mathscr{E}$ by giving to each trader in $\mathscr{E}$ the joint information of all the traders in $\mathscr{E}$, i.e., for all $t \in T, \mathscr{T}_{t}^{*}=\bigvee_{i=1}^{n} \mathscr{T}_{i}$, and leaving the rest of his characteristics unchanged. Note that in $\mathscr{E}^{*}$ all traders have the same information (i.e., $\mathscr{E}^{*}$ is an economy with symmetric information). For each $t \in T$, we denote by $M_{t}^{*}$ the set of all $\mathscr{F}_{t}{ }^{*}$-measurable functions from $\Omega$ to $\Re_{+}^{l}$. Let $p: \Omega \rightarrow \Re_{+}^{l}$ be a price system. In the economy $\mathscr{E}^{*}$ the budget set of $t \in T$ with respect to $p$ is

$$
B^{*}(p, t)=\left\{x \mid x \in M_{t}^{*}, \sum_{\omega \in G} p(\omega) \cdot x(\omega) \leq \sum_{\omega \in G} p(\omega) \cdot \mathbf{e}(t, \omega)\right\} .
$$

A competitive equilibrium of $\mathscr{E}^{*}$ (in the sense of Radner) is now defined as in Section 2.

Proposition 4.1. Every competitive allocation of $\mathscr{E}^{*}$ is in the weak fine bargaining set of $\mathscr{E}$. 
Proof. It is easy to see that an economy $\mathscr{E}$ as defined in Section 2 satisfies the assumptions of Theorem C in Einy, Moreno and Shitovitz (1998), which establishes that the set of competitive allocations of $\mathscr{E}^{*}$ coincides with the weak fine core of $\mathscr{E}$. Since the weak fine core is a subset of the weak fine bargaining set, Proposition 4.1 readily follows from this result.

As the following example shows, the analog of Theorem C in Einy, Moreno and Shitovitz (1998) for the weak fine bargaining set does not hold: there are allocations in the weak fine bargaining set that are not competitive allocations of $\mathscr{E}^{*}$. For the analysis of the example we need some notation and a lemma which is interesting on its own.

If $\mathscr{E}$ is an economy and $S$ is a coalition with $\mu(S)>0$, we denote by $\mathscr{E}_{S}$ the restriction of $\mathscr{E}$ to $S$; that is, $\mathscr{E}_{S}$ is an economy for which the space of traders is $\left(S, \Sigma_{S}, \mu_{S}\right)$, where $\Sigma_{S}=\{Q \mid Q \in \Sigma, Q \subset S\}$, and $\mu_{S}$ is the restriction of $\mu$ to $\Sigma_{S}$.

Lemma 4.2. Let $\mathscr{E}$ be an economy. Assume that $(S, \mathbf{y})$ is a justified weak fine objection to a weak fine allocation $\mathbf{x}$ in $\mathscr{E}$. Then the restriction of $\mathbf{y}$ to $S \times \Omega$ is a competitive allocation of $\mathscr{E}_{S}^{*}$.

Proof. Assume by way of contradiction that the restriction $\hat{\mathbf{y}}$ of $\mathbf{y}$ to $S \times \Omega$ is not competitive in $\mathscr{E}_{S}^{*}$. Then by Theorem C of Einy, Moreno and Shitovitz (1998), $\hat{\mathbf{y}}$ is not in the weak fine core of $\mathscr{E}_{S}$. Therefore $\hat{\mathbf{y}}$ has a weak fine objection $(Q, \hat{\mathbf{z}})$ in $\mathscr{E}_{S}$. Let $\mathbf{z}$ be an extension of $\hat{\mathbf{z}}$ to an assignment in $\mathscr{E}$. As $Q \subset S,(Q, \mathbf{z})$ is a weak fine counterobjection to $(S, \mathbf{y})$ in $\mathscr{E}$. But this contradicts our assumption that $(S, \mathbf{y})$ is a weak fine justified objection to $\mathbf{x}$ in $\mathscr{E}$.

Example 4.3. Consider an economy $\mathscr{E}$ in which the commodity space is $\Re_{++}$, and the set of traders is $([0,3], \mathscr{B}, \mu)$, where $\mathscr{B}$ is the $\sigma$-field of Borel subsets of $[0,3]$ and $\mu$ is the Lebesgue measure. The space of states of nature is $\Omega=$ $\left\{\omega_{1}, \omega_{2}\right\}$. All traders have the same utility function, given for $(\omega, x) \in \Omega \times \Re_{++}$ by

$$
u(\omega, x)=\ln x .
$$

The initial assignment is $\mathbf{e}(t, \omega)=2$, for all $(t, \omega) \in T \times \Omega$. Let $T_{1}=[0,1], T_{2}=$ $(1,2]$, and $T_{3}=(2,3]$. The information partition of a trader $t \in T_{1} \cup T_{2}$ is $\Pi_{1}=\Pi_{2}=\{\Omega\}$, and that of the traders $t$ in $T_{3}$ is $\Pi_{3}=\left\{\left\{\omega_{1}\right\},\left\{\omega_{2}\right\}\right\}$. The priors of the traders in $T_{1}, T_{2}$, and $T_{3}$ are, respectively, $q_{1}=\left(\frac{1}{4}, \frac{3}{4}\right), q_{2}=\left(\frac{3}{4}, \frac{1}{4}\right)$, and $q_{3}=\left(\frac{1}{2}, \frac{1}{2}\right)$. We construct a weak fine bargaining set allocation of $\mathscr{E}$ which is not competitive in $\mathscr{E}^{*}$.

Define an assignment $\mathbf{x}: T \times \Omega \rightarrow \Re_{++}$by

$$
\mathbf{x}\left(t, \omega_{1}\right)= \begin{cases}1 & t \in T_{1} \\ 2.55 & t \in T_{2} \\ 2.45 & t \in T_{3},\end{cases}
$$

and 


$$
\mathbf{x}\left(t, \omega_{2}\right)= \begin{cases}2.55 & t \in T_{1} \\ 1 & t \in T_{2} \\ 2.45 & t \in T_{3}\end{cases}
$$

Then $\mathbf{x}$ is a weak fine allocation in $\mathscr{E}$. We show that $\mathbf{x}$ is in the weak fine bargaining set of $\mathscr{E}$, but it is not competitive in $\mathscr{E}^{*}$. Assume, by way of contradiction, that $(S, \mathbf{y})$ is a justified weak fine objection to $\mathbf{x}$ in $\mathscr{E}$. Then by Lemma 4.2 the restriction $\hat{\mathbf{y}}$ of $\mathbf{y}$ to $S \times \Omega$ is a competitive allocation in $\mathscr{E}_{S}^{*}$. Now if $\mu\left(S \cap T_{3}\right)=0$, then we must have that $\hat{\mathbf{y}}(t, \omega)=\mathbf{e}(t, \omega)$ for all $(t, \omega) \in S \times \Omega$. As $h_{t}(\mathbf{e}(t, \cdot))<h_{t}(\mathbf{x}(t, \cdot))$ for all $t \in T_{1} \cup T_{2}$, this leads to a contradiction. Assume that $\mu\left(S \cap T_{3}\right)>0$. Let $p$ be a price system such that $(p, \hat{\mathbf{y}})$ is a competitive equilibrium of $\mathscr{E}_{S}^{*}$. Then $p\left(\omega_{1}\right)>0$ and $p\left(\omega_{2}\right)>0$. Without loss of generality assume that $p\left(\omega_{2}\right)=1$, and denote $r=p\left(\omega_{1}\right)$. Then the first order conditions for utility maximization imply that for almost all $t \in S$,

$$
\hat{\mathbf{y}}\left(t, \omega_{1}\right)= \begin{cases}\frac{1}{2}\left(1+\frac{1}{r}\right) & t \in T_{1} \\ \frac{3}{2}\left(1+\frac{1}{r}\right) & t \in T_{2} \\ 1+\frac{1}{r} & t \in T_{3},\end{cases}
$$

and

$$
\hat{\mathbf{y}}\left(t, \omega_{2}\right)= \begin{cases}\frac{3}{2}(1+r) & t \in T_{1} \\ \frac{1}{2}(1+r) & t \in T_{2} \\ 1+r & t \in T_{3}\end{cases}
$$

Since $\mu(S)>0$ and $(p, \hat{\mathbf{y}})$ is a competitive equilibrium of $\mathscr{E}_{S}^{*}$, we have

$$
\left(1+\frac{1}{r}\right)(1+r) \leq \frac{16}{3}<(2.45)^{2}
$$

Therefore for almost all $t \in S \cap T_{3}$ we have

$$
h_{t}(\mathbf{y}(t, \cdot))=\frac{1}{2} \ln \left[\left(1+\frac{1}{r}\right)(1+r)\right]<\frac{1}{2} \ln (2.45)^{2}=h_{t}(\mathbf{x}(t, \cdot)) .
$$

As $\mu\left(S \cap T_{3}\right)>0$, this contradicts the assumption that $(S, \mathbf{y})$ is a weak fine objection to $\mathbf{x}$.

The above argument shows that if, in particular, $\mathbf{z}$ is a competitive allocation of $\mathscr{E}^{*}$, then for almost all $t \in T_{3}$

$$
h_{t}(\mathbf{z}(t, \cdot))<h_{t}(\mathbf{x}(t, \cdot))
$$

Therefore $\mathbf{x}$ is not a competitive allocation of $\mathscr{E}^{*}$. Note that the last inequality implies that the informed traders (i.e., the traders in $T_{3}$ ) are better off in $\mathbf{x}$ than in any competitive allocation of $\mathscr{E}^{*}$. 


\section{References}

Allen, B.: Market games with asymmetric information and non-transferable utility: representations results, and the core. Mimeo, University of Pennsylvania (1991)

Aumann, R.J.: Markets with a continuum of traders. Econometrica 32, 39-50 (1964)

Aumann, R.J.: Values of markets with a continuum of traders. Econometrica 43, 611-646 (1975)

Aumann, R.J. and Maschler, M.: The bargaining set for cooperative games. In: Dresher, M., Shapley, L.S., Tucker, A.W. (eds.) Advances in game theory, Annals of Mathematical Studies, no. 52, pp. 443-476. Princeton, NJ: Princeton University Press 1964

Debreu, G.: Theory of value, New Haven: Yale University Press 1959

Einy, E., Moreno, D., Shitovitz, B.: Existence and equivalence of competitive and core allocations in large exchange economies with differential information. University Carlos III de Madrid, WP 98-71 (1998)

Einy, E., Moreno, D., Shitovitz, B.: Fine value allocations in large exchange economies with differential information. Mimeo (1999)

Einy, E., and Shitovitz, B.: Equivalence of value and competitive allocations in large exchange economies with differential information. Mimeo (1998)

Koutsougeras, L.C., and Yannelis, N.C.: Incentive compatibility and information superiority of the core of an economy with differential information. Economic Theory 3, 195-216 (1993)

Krasa, S., and Yannelis, N.C.: The value allocation of an economy with differential information. Econometrica 62, 881-900 (1994)

Maschler, M.: An advantage of the bargaining set over the core. Journal of Economic Theory 13, 184-194 (1976)

Maschler, M.: The bargaining set, kernel, and nucleolus. In: Aumann, R.J., Hart, S. (eds.) Handbook of game theory, vol. 1, pp. 591-667. Amsterdam: Elsevier 1992

Mas-Colell, A.: An equivalence theorem for a bargaining set. Journal of Mathematical Economics 18, 129-139 (1989)

Radner, R.: Competitive equilibrium under uncertainty. Econometrica 36, 31-58 (1968)

Radner, R.: Equilibrium under uncertainty. In: Arrow, K.J., Intriligator, M.D. (eds.) Handbook of Mathematical Economics, vol. II. Amsterdam: North Holland 1982

Wilson, R.: Information, efficiency, and the core of an economy. Econometrica 40, 807-816 (1978)

Yannelis, N.C.: The core of an economy with differential information. Economic Theory 1, 183-198 (1991) 